\title{
O progresso das cerâmicas no uso odontológico: uma revisão de literatura
}

The progress of ceramics in dental use: a review of literature

El progreso de la cerámica en el uso dental: una revisa de literatura

José Milton de Aquino e Silva Neto ${ }^{1 *}$, Morgana Lorena Paulo dos Santos ${ }^{1}$, Arlette Murgado Trujillo1, João Vitor Brasil Alves Rodrigues Almeida Barros ${ }^{1}$, Júllian Karen Bezerra dos Santos ${ }^{1}$, Tayguara Cerqueira Cavalcanti', Michelle Leão Bittencourt Brandão Medeiros ${ }^{1}$.

\section{RESUMO}

Objetivo: Apresentar e revisar o progresso nas últimas décadas das cerâmicas utilizadas pelo Cirurgião Dentista (CD). Revisão bibliográfica: Nos dias atuais a cerâmica utilizada pelo CD são uma das ferramentas que mais se destacam quando se refere a produção de dentes artificiais, tornando-se referência na produção de procedimentos inlays, onlays, overlays, pinos, núcleos intra-canais e até mesmo implantes dentais, isso ocorre devido as inúmeras qualidades favoráveis que elas possuem, sendo destacado os coeficientes de expansões térmicas parecido com os dentes naturais, sua translucidez, equilíbrio químico, fluorescência, resistência à abrasão e à compressão, beneficiando dessa forma, para uma longevidade dos reparos estabelecidos. Considerações Finais: Para a obtenção de um resultado satisfatório o CD precisará classificar e qualificar, de maneiras distintas, as particularidades de cada paciente para só assim poder garantir uma maior longevidade e sucesso do tratamento restaurador, visto que a exigência para a obtenção de um resultado final eficiente utilizando as cerâmicas são inúmeros.

Palavras-chave: Cerâmica, Porcelana dentária, Próteses e implantes, Restaurações intracoronárias.

\begin{abstract}
Objective: Present and review the progress in the last decades of the ceramics used by the Dentist Surgeon (CD). Literature review: Nowadays, the ceramics used by the CD are onde of the most outstanding tools when it comes to the production of artificial teeth, becoming a reference in the production of inlays, onlays, overlays, pins, intra-canal cores and even dental implants. This is due to the numerous favorable qualities that they possess, being highlighted the coefficients of thermal expansion similar to natural teeth, their translucency, chemical balance, fluorescence, resistance to abrasion and compression, thus benefiting for a longevity of established repairs. Final Considerations: In order to obtain a satisfactory result, the CD will need to classify and qualify, in different ways, the particularities of each patient in order to guarantee the longevity and success of the restorative treatment, since the requirement to obtain an efficient final result using the ceramics are numerous.
\end{abstract}

Keywords: Ceramics, Dental porcelain, Protheses and implants, Inlays.

\section{RESUMEN}

Objetivo: Presentar y revise el progreso en las últimas décadas de la cerámica utilizada por el Dentista Cirujano (CD). Revisión bibliográfica: Hoy en día, la cerámica utilizada por el CD es una de las herramientas más destacadas en lo que respecta a la producción de dientes artificiales, convirtiéndose en una referencia en la producción de incrustaciones, inlays, onlays, overlays, alfileres, núcleos intracanales e incluso implantes

${ }^{1}$ Centro Universitário CESMAC (CESMAC), Maceió-Alagoas. *E-mail: Milton_neto_166@hotmail.com 
dentales. Esto se debe a las numerosas cualidades favorables que poseen, destacando los coeficientes de expansión térmica similares a los dientes naturales, su translucidez el equilibrio químico, la fluorescencia, la resistencia a la abrasión y la compresión, lo que beneficia la longevidad de las reparaciones establecidas. Consideraciones finales: Para obtener un resultado satisfactorio, el CD deberá clasificar y calificar, de diferentes maneras, las particularidades de cada paciente para garantizar la longevidad y el éxito del tratamiento restaurador, ya que el requisito para obtener un resultado final eficiente utilizando la cerámica es numerosa.

Palabras clave: Cerámica, Porcelana dental, Prótesis e implantes, Incrustaciones.

\section{INTRODUÇÃO}

As primeiras coroas e inlays de cerâmicas feldspáticas fundidas foram introduzidas na odontologia por Land em 1886 (CALIXTO R e MASSING N, 2015). Em 1894, surgiram novas formas de manuseio da cerâmica a partir da invenção do forno elétrico, e em 1903 com a utilização de coroas de jaqueta de porcelana, as cerâmicas por fim, entraram na odontologia restauradora (SHRIVASTAVA S, et al., 2014).

Desde então, mudanças na fabricação, composição, propriedades, indicações e modo de uso, levaram a uma cerâmica dental altamente estética e biocompatível, porém ainda fraca em algumas situações, principalmente quando usada em espessura muito delgada (CALIXTO R e MASSING N, 2015).

Nos dias atuais a crescente demanda por uma busca de padronização estética e harmoniosa dos elementos dentais fez com quer os Cirurgiões Dentistas (CD) buscassem empregar materiais restauradores em seus consultórios que expressassem boa forma mecânica e apresentável, utilizando por sua vez cada vez mais os materiais compostos por cerâmica.

Desta forma, pode ser observado que seus componentes presentes nas estruturas possuem forma inorgânicas formada de oxigênio dos quais podem existir alguns princípios metálicos ou semi-metálicos agregados (FERREIRA HA, et al., 2016).

As cerâmicas apresentam inúmeras propriedades benéficas, quando utilizadas como sucessor de elementos dentais naturais, possuindo bom coeficiente de expansão térmica e estabilidade química, translucidez parecido com dos dentes, bastante resistente as abrasões e à compressões. Contudo, para se obter um bom resultância no padrão esperado deve-se ter atenção com a integridade marginal, a forma que se caracteriza a superfície, respeitando toda a anatomia e por fim, porém não o menos importante a sua tonalidade da cor (CALIXTO R e MASSING N, 2015).

A cerâmica assemelha-se a estrutura dental, com isso permite uma excelente adaptação marginal e interrelação com os elementos que compõem o periodonto, auxiliando assim, melhor tempo de longevidade do tratamento restaurador estabelecido (SHRIVASTAVA S, et al., 2014).

É importante obter atenção quando e qual a técnica utilizar pois o processo de resistência flexural e a sua tenacidade são sinais para a escolha da cerâmica adequada em cada caso particular, visto que em regiões de elementos dentais posteriores e caninos, do qual recebem maior força de tensão, precisam de cerâmicas que apresentem elevados rendimentos dessas propriedades (CALIXTO R e MASSING N, 2015).

No ato do restabelecimento da reabilitação do paciente é importante levar em consideração as funções, as formas biológicas e sua parte estética, tendo como objetivo a durabilidade do procedimento. O CD deve possuir habilidade e atenção nas espessuras que irá ser montada a peça e respeitar a cor (CALIXTO R e MASSING N, 2015).

O sucesso depende bastante da adaptação feita no ato do procedimento reabilitador, não deixando presença de infiltração ou cárie secundária, obtenção de um bom material adesivo, analisar os agentes cimentantes que irá ser utilizado, além de analisar as possíveis instabilidades existentes (BISPO LB, 2015).

Assim sendo, este trabalho tem como principal fundamento, através de uma revisão de literatura, colaborar com a escolha e recomendação dos materiais cerâmico com o compreendimento da forma clínica encontrada em cada caso, exemplificando as expectativas que podem ser geradas na escolha do mesmo. 


\section{REVISÃO BIBLIOGRÁFICA}

Cerâmicas são sólidos inorgânicos e não metálicos originados do aquecimento e resfriamento dos componentes na sua forma bruta, individualmente ou misturado. As cerâmicas odontológicas são originadas de outras técnicas ou apresentam alguns componentes orgânicos em sua composição podendo conter uma estrutura totalmente ou parcialmente cristalina ou podendo ser amorfa como o vidro (MAGNO P e BELSER U, 2012).

Elas são materiais designados para reproduzir prótese dental, que são usados para devolver dentes perdidos ou destruídos e também conhecida como porcelana dental, são materiais semelhantes ao dente natural, por possuírem propriedades ópticas, excelente estética e alta taxa de longevidade, com indicações de uso muito amplas que vão desde pequenos fragmentos cerâmicos e restaurações indiretas inlays e onlays às coroas unitárias, próteses parciais fixas extensas e protocolos completos de implantes(HELVEY GA, 2014).

Seguindo o mesmo princípio das resinas compostas, as cerâmicas também são compósitas, ou seja, são formados por vários componentes. Tendo como matriz o vidro, levemente ou fortemente infiltrados a altas temperaturas com partículas de vidro ou cristalinas (MCLAREN EA e FIGUEIRA J, 2015).

Os sistemas totalmente cerâmicos surgiram com o objetivo de eliminar as estruturas metalocerâmicas (infraestruturas de metal cobertas com porcelana), resultando numa melhor funcionalidade e estética decorrente da distribuição da reflexão da luz (MAGNO P e BELSER U, 2012).

Possui a capacidade de mimetizar tecidos dentais, durabilidade, ampla aceitação (profissionais e pacientes), natureza refratária, inércia química, friabilidade, susceptibilidade à fratura, baixa resistência à tração, tornam a cerâmica um material bem conhecido, além do fato de se apresentarem como uma solução mais conservadora, preservando estrutura dental e devolvendo cor, estética, forma, posição e textura (HELVEY GA, 2014).

Nos últimos 10 anos, ocorreu um aumento no número de materiais cerâmicos para restaurações totalmente cerâmicas, e cada um deles, de maneira diferente, tenta melhorar as suas propriedades mecânicas sem perder na estética. Para selecionar adequadamente o material para determinadas situações clínicas, deve-se saber algumas características desses materiais, além de preservar estrutura dentária e observar requisitos necessários para manutenção do tratamento reabilitador, design do sorriso e estética (HELVEY GA, 2014).

Apesar das dificuldades em agrupar os diversos sistemas cerâmicos, existe uma variedade de formas para classificar esses materiais e algumas classificações vêm sendo propostas (CALIXTO R e MASSING N, 2015). Classificar as cerâmicas de acordo com sua composição, método de processamento, resistência, temperatura de fusão, translucência, indicações clínicas (anteriores ou posteriores, coroas totais ou parciais), cimentação (adesiva ou tradicional) e sensibilidade de superfície, podem facilitar a avaliação para a escolha do material para cada caso (SHRIVASTAVA S, et al., 2014).

\section{Cerâmicas Vítreas (feldspáticas)}

Fabricadas pelo método da estratificação, são as mais translucentes e mais fracas também, conferindo naturalidade à coroa, consideradas as cerâmicas livres de metal mais conservadoras, necessitando apenas de uma espessura de 0,2 a $0,3 \mathrm{~mm}$. Ideal para casos onde haja uma quantidade significativa de esmalte remanescente mais ou menos $50 \%$ e remanescente dental sadio, e $70 \%$ ou mais da margem seja também em esmalte e precisam de polimento ou glazeamento para serem finalizadas. São indicadas para inlays, onlays, facetas, dentes anteriores e premolares, ocasionalmente molares, coroas parciais e totais (CALIXTO R e MASSING N, 2015).

\section{Cerâmicas reforçadas}

Para controlar efeitos ópticos e mimetizar naturalmente esmalte e dentina, fabricantes adicionaram pequenas quantidades de partículas de carga e, quanto maior a quantidade de partículas, melhores propriedades mecânicas e menor a estética. O reforço da porcelana para que ela conseguisse suportar os esforços pode ser alcançado de diferentes formas: fusão ao metal, reforçadas: desenvolvidas para substituir a infraestrutura metálica por outra de cerâmica opaca, surgindo em 1960, com a invenção da alumina. Cimentada com óxido de zinco, que não tem adesão à coroa de porcelana e, apesar de serem um avanço, 
seu uso estava restrito a próteses unitárias e coroas e, são contraindicadas para regiões posteriores. A adição de óxidos às cerâmicas tem a intenção de aumentar a resistência flexural das mesmas. Necessitam de espessura de trabalho de aproximadamente $0,8 \mathrm{~mm}$ (SHRIVASTAVA S, et al., 2014).

Os vidros ceramizados (fundidos, injetados ou prensados), foram obtidos através da cristalização seletiva e controlada de alguns vidros. São sistemas livres de metal que possuem características de vidros e de cerâmicas, melhorando assim sua estética [30], usados em infraestrutura totalmente cerâmicas através da técnica injetada. [12].

Em 2002, a Sirona Dental® introduziu no mercado um sistema o sistema Cerec in Lab®, específico para laboratório, que utiliza a leitura do troquel com laser na mesma unidade compacta em que a fresagem para fabricação de copings e infraestruturas de até 3 elementos é feita (DINATO JC, et al., 2014).

\section{Dissilicato de lítio}

Surgiram em 1999, com o objetivo de ampliar a indicação das cerâmicas para a região de $2^{\circ}$ molar. Apresentam fase vítrea amorfa com estrutura porosa, composta por sílica, dióxido de lítio, alumina, óxido de potássio e pentóxido de fósforo (SILVA W, et al., 2015). Contêm cerca de 70\% de cristais de dissilicato de lítio dispersos na matriz vítrea com tratamento para nucleação e crescimento dos cristais melhorando suas propriedades físicas e mecânicas, sem comprometer as propriedades ópticas, e gerando um stress de compressão em torno deles. São aproximadamente quatro vezes mais resistentes que as feldspáticas (DINATO JC, et al., 2014).

\section{Alumina infiltrada por vidro}

Os cristais de alumina sob três formas distintas: grãos de 10 a $12 \mu \mathrm{m}$ de comprimento e 2,5 a $4 \mu \mathrm{m}$ de largura, partículas de 1 a $4 \mu \mathrm{m}$ de diâmetro e grãos esféricos de menos de $1 \mu \mathrm{m}$ de diâmetro inseridos na cerâmica, numa proporção de $50 \%$, melhoram a resistência flexural da mesma e diminuem sua translucidez, devido à limitação de transmissão da luz pelos mesmos. As cerâmicas reforçadas com óxidos metálicos, as cerâmicas aluminizadas, originaram uma nova classe de sistemas cerâmicos e surgiram devido à busca por uma maior resistência estrutural, com intenção de ter o dobro da resistência à fratura das feldspáticas convencionais (ANUSAVICE KJ et al., 2013).

Usam a técnica slip-casting, pasta de óxido de alumínio $\left(\mathrm{Al}_{2} \mathrm{O}\right)$ é aplicada no molde refratário e é sintetizado, produzindo estrutura porosa de partículas de alumina que é infiltrada com vidro de lantânio na segunda queima, partículas essas, que foram adicionadas (vidro aluminosilicato de lantânio) no intuito de eliminar a porosidade, aumentar a força e limitar a propagação de fissuras, melhorando as tensões de compressão quando fossem introduzidas forças maiores às cerâmicas (DINATO JC, et al., 2014).

Avanços no processo levaram a um material com alto conteúdo de alumina, com porosidade diminuída, densamente sintetizada, utilizadas também para sistemas de desenho assistido por computador (CAD) e manufatura assistida por computador (CAM) são capazes de suportar cargas de até $750 \mathrm{MPa}$, levando sua indicação também para dentes posteriores (BISPO LB, 2015).

Criam-se poros na matriz que é formada num bloco e depois processada usando tecnologia CAD/CAM, onde o material é infiltrado por ação capilar, sendo atualmente no mercado, substituído por material com $100 \%$ de matriz cristalina. Possuem indicação para coroas anteriores, apesar de não possuírem boas propriedades ópticas, posteriores, próteses parciais fixas de três elementos na região anterior e confecção de núcleos cerâmicos, na confecção de abutments personalizados para implantes, prótese sobre implantes na região posterior (CHUN EP, et al., 2017).

\section{Zircônia infiltrada por vidro}

É opaca e tem pouca translucência e são utilizadas para coroas posteriores e próteses parciais fixas posteriores. Próteses parciais fixas de 3 ou 4 elementos em região anterior e próteses sobre implantes. Podem ser utilizados em copings e abutments (infraestruturas de restaurações cerâmicas), na mesma espessura de um coping metálico, já que por possuírem óxidos em sua composição, essas cerâmicas possuem maior resistência e por apresentarem alto conteúdo cristalino, elas aparecem opacas à luz visível, com baixa translucidez necessitando de uma cerâmica de cobertura mais estética (VARGAS MA, 2011). Como as cerâmicas podem também ser cimentadas sobre remanescentes dentais vitais, deve-se lembrar de 
utilizar um cimento que impeça que a umidade natural oriunda dos túbulos dentinários entre em contato com a superfície da zircônia (MATTEI FP e ALEXANDRE MC, 2011).

Um dos maiores valores de tenacidade entre as cerâmicas, é encontrado nas cerâmicas infiltradas com zircônia gerando, porém, um sistema bastante opaco e com indicações limitadas para a região posterior (prótese fixa de três elementos e unitárias).

Já o sistema Procera - Nobel Biocare, apresenta altíssima concentração de óxido de alumínio altamente purificado e densamente sinterizado constituído por mais de $99,5 \%$ de alumina $\left(\mathrm{Al}_{2} \mathrm{O}_{3}\right)$, indicado para subestruturas de próteses cerâmicas utiliza a tecnologia CAD/CAM (Computer-Aided Design/ ComputerAssisted Machining) que é um processo industrial computadorizado, que digitaliza a moldagem realizada pelo cirurgião-dentista, usando um dispositivo de scanner e um computador que transforma o dado do molde digitalizado em um dado, que será utilizado para fabricar a restauração (NAUTIYAL A, et al., 2015).

Em casos de peças cerâmicas espessas (acima de 1,3mm) ou em coroas com copings de zircônia/alumina/metal, pouca ou nenhuma influência da cor ou do substrato dentário pode-se observar. Nos casos de laminados (espessuras abaixo de $0,5 \mathrm{~mm}$ ), tanto o substrato como o agente cimentante, influenciam bastante no resultado final e na estabilidade de cor uma vez que o "envelhecimento" fisiológico do substrato ou alguma pigmentação extrínseca advinda da face palatina do mesmo podem alterar a cor.

Entretanto ainda permanecem algumas características que dificultam suas indicações na clínica, tais como: opacidade, dificuldade na cimentação já que não pode ser condicionado e envelhecimento promovido pelo contato com a umidade. São sinterizados em forno próprio para sistema após usinagem (NAUTIYAL A, et al., 2015).

\section{Zircônia}

A zircônia estabilizada por ítrio (Y-TZP) surgiu no intuito de diminuir a propagação de trincas, controlando a expansão do volume. São mais versáteis por possuírem boas propriedades mecânicas, estética, biocompatibilidade, alta resistência à fratura e baixo módulo de elasticidade (ANUSAVICE KJ et al., 2013).

Conhecido como mecanismo de tenacificação (transformation toughening), transformação e retardo na propagação de trincas, o aumento do volume dos cristais, causado por estímulos (impactos, carga), cria uma compressão na rachadura com a intenção de neutralizar a tensão externa, impedindo a propagação das trincas na superfície protética. Apesar de não impedir progressão da fratura, ele torna mais difícil de a mesma acontecer (GRACIS S, et al., 2015).

A sua classificação pode ser feita de acordo com o grau de estabilização microestrutural da mesma: completamente estabilizada (FSZ), parcialmente estabilizada (PSZ), permitem manipulação pelo técnico ou unidade de fresagem com dureza menor, antes da sinterização, e policristalina na forma tetragonal (TZP) que são as cerâmicas dentais, mais comumente sendo as estabilizadas por ítrio (Y-TZP), necessitam de maior tempo de fresagem ( 2 a 4h), geram maior desgaste das fresas, promovem lascamentos e trincas na superfície, gerando um comprometimento da longevidade da restauração (CONRAD HJ, et al., 2007).

Y-TZP HT são zircônias estabilizadas por ítrio fabricadas com alta translucência para ser utilizada em sistemas CAD/CAM, com indicações para usos monolíticos, as quais podem ser ajustadas nas faces oclusais e proximais durante a aplicação clínica utilizando-se pontas diamantadas (CALIXTO R e MASSING N, 2015).

Porém, esses ajustes podem levar ao início de propagação de cracks quando submetidos a stress, mas esse risco pode ser minimizado com a utilização de kits adequados de polimento com pontas diamantadas de forma adequada, originando uma superfície mais homogênea, a qual pode ser comparada ao glazeamento (GRACIS S, et al., 2015).

Inicialmente criada para cobertura de coroas, atualmente é o material cerâmico mais resistente, devido à alta resistência flexural e tenacidade à fratura provenientes de sua composição altamente cristalina e sem componentes vítreos, podendo ser utilizadas como infraestrutura de próteses fixas unitárias, múltiplas, como material monolítico, além da confecção de pontes livres de metal e na confecção de coroas monolíticas (MATTEI FP e ALEXANDRE MC, 2011).

100\% zircônia cristalinas: inicialmente baseadas em alumina, com boa indicação para coroas unitária, mais recentemente à base de zircônia são mais translucentes, usadas na forma monolítica em posteriores. 
Indicadas também para regiões de perdas significantes, presença de flexão ou stress, coroas totais posteriores, próteses parciais fixas, quando há dificuldade com procedimentos adesivos como margens subgengivais (KRISHNA JV, et al., 2009).

\section{Quanto à técnica de confecção}

Podem ser classificadas em: condensação sob troquel refratário; injetadas (cera perdida); pó e líquido, maquiagem, estratificação; slip casting; CAD/CAM (MATTEI FP e ALEXANDRE MC, 2011).

\section{Injetados (cera perdida) ou prensada}

Técnica introduzida nos anos 80 , permitindo ao técnico criar a restauração em cera, a qual, através da técnica da cera perdida, a cerâmica, na sua forma plástica é submetida à pressão hidrostática, escoam para o interior do molde, fazendo apenas a aplicação da cerâmica de cobertura após a remoção da mesma de dentro do molde. Cerâmicas com grande quantidade de leucita foram inicialmente usadas nesse processo. Em 2006, o dissilicato de lítio foi o segundo material a utilizar este método (SKRIPNIK NN, 2016).

\section{Estratificação}

É o método convencional sendo construída sob troquel metálico ou de cerâmica, incorporando-se o pó e o líquido manualmente com a ajuda de um pincel ou espátula. Em algumas fases da fabricação, a cerâmica inserida é eliminada no vácuo a uma determinada temperatura, removendo a umidade e condensando a cerâmica num processo chamado de sinterização, onde ocorre fusão no ponto de contato das partículas, resultando num aumento da densidade quando cerâmica ou vidro alcancem a temperatura de fusão. Ex: cerâmicas feldspáticas e à base de fluoropatitas (GRACIS S, et al., 2015).

\section{Maquiagem}

Ocorre a individualização das restaurações injetadas com diferentes pigmentos de cor, através da aplicação de camadas bem finas dos mesmos após o polimento e fixadas à temperatura de $770^{\circ} \mathrm{C}$, necessitando muitas vezes de várias queimas para chegar ao estágio final para assegurar que a escovação e polimentos não irão removê-los (NAUTIYAL A, et al., 2015).

\section{CAD/CAM}

Usinagem ou torneamento computadorizado. Introduzido em meados dos anos 90, por Nobel Biocare, o primeiro produto totalmente cerâmico produzido com CAD/CAM, na qual o núcleo era constituído por $99,9 \%$ de alumina, onde a cerâmica feldspática era depositada em camadas. O uso da tecnologia CAD/CAM, permite a fabricação através de escaneamento e fresagem das restaurações unitárias ou múltiplas através do uso de computador. O sistema é composto por 3 componentes funcionais: captura de dados ou escaneamento para capturar e salvar dados, o CAD faz o design da restauração e o CAM fabrica a restauração (CALAMIA JR e CALAMIA CS, 2007).

Dois métodos são utilizados: eletrodeposição (deposição em camadas do material sob a forma de pó, também chamada de prototipagem rápida), e a mais comum, que é através da fresagem de um bloco sólido de cerâmica que posteriormente podem ser submetidas a um processo de sinterização, tornando essa estrutura mais densa e aumentando sua resistência flexural para 1.000 a 1.200 Mpa. São utilizadas cerâmicas baseadas em sílica, cerâmicas infiltradas, cerâmicas de dissilicato de lítio e cerâmicas óxidas de alta performance.

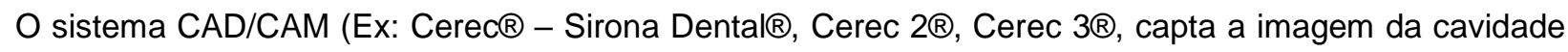
bucal através de microcâmera, que realiza leitura óptica de todo o preparo, o qual deve ser previamente coberto por dióxido de titânio pulverizado para que a luz se difunda de maneira uniforme, processando a imagem na unidade CAD e planeja e executa a restauração com auxílio do computador (CAM). A restauração é executada pela técnica de fresagem do bloco cerâmico com ponta diamantadas e disco para desgaste (CEREC 2®), duas pontas na unidade modular (CEREC $3 \AA$ ) (TRINDADE AC, et al., 2016).

Depois de ajustes e polimento inicial faz-se a c3aracterização com pigmentos e o glazeamento. Os sistemas CAD/CAM têm se tornado comum entre profissionais e pacientes que buscam por um tratamento livre de metal, permitindo a junção da estética com a resistência, materiais usados diferem na composição, indicação, propriedades, e métodos de processamento. Substituem o processo de cera perdida. CAD pode 
ser dividido em duas etapas, através de scanner, software e máquina de fresadora e impressão digital em 3D. Procedimento intraoral e laboratorial. É composto por scanner para copiar estrutura em boca ou modelos de gesso, e um computador que receberá os dados e gerará imagem tridimensional. São indicados para coroas totais, inlays, onlays, facetas, pilares personalizados, pontes fixas, copings e infraestruturas de pontes (NAUTIYAL A, et al., 2015).

Deve-se lembrar sempre que além da escolha adequada e respeito ao protocolo restaurador, existem vários fatores para os progressos envolvidos no sucesso da reabilitação com cerâmicas como: adequado preparo dental, adaptação marginal, moldagem, interação com tecidos moles e escolha do ceramista e que mostrar que bons resultados não advêm exclusivamente de boa escolha do tipo da cerâmica utilizada, mas também da seleção correta do material, técnica de execução e habilidade profissional (TRINDADE AC, et al., 2016).

\section{CONSIDERAÇÕES FINAIS}

Os avanços das cerâmicas na odontologia estão sendo bastante elucidados, surgindo cada vez mais técnicas cruciais para o melhor desempenho da função e respectivamente da sua estética, inúmeros procedimento cerâmico vem sendo utilizados e disponíveis no mercado. Cada um possui suas particularidades apresentando tanto vantagens como desvantagens, no decorrer dos estudos feitos através de artigos científicos, pode-se concluir que cabe ao Cirurgião Dentista escolher a técnica mais indicada para cada paciente, porém é necessário ressaltar que alguns sistemas novos que estão sendo implantados possuem pouco tempo de utilização, sendo relevante tanto ao paciente quando ao profissional fazer acompanhamentos, principalmente quando se comparada as coroas metalocerâmicas, que possuem um estudo longitudinal bastante expressivo. Indica-se, portanto, que o profissional seja qualificado e possua uma boa competência técnica e cientifica para o desenvolvimento de tal procedimento.

\section{REFERÊNCIAS}

1. ANUSAVICE KJ, et al. Materiais dentários, 12ed, Elsevier, Rio de Janeiro, 2013; p. 619.

2. BISPO LB. Cerâmicas odontológicas: vantagens e limitações da zircônia. Rev Bras.Odontol. v. 76, n 1/2, 2015; p. 24 29.

3. CALIXTO R, MASSING N. Longevidade das restaurações cerâmicas anteriores. Parte 1, Rev. Dental Press Estética, Araraquara, 2015; p. 18-28.

4. CHUN EP, et al. Microstructural analysis and reliability of monolithic zircônia after simulated adjustement protocols, Dent Mater. 2017.

5. CONRAD HJ, et al. Current ceramic Materials and Systems with Clinical Recommendations: A systematic review, J.Prosthet Dent. 2007; p.389.

6. DINATO JC, et al. Sistema CAD/CAM - substituindo o processo de cera perdida na prática clínica com maior precisão, resistência e menor custo, ProteseNews. 2014; p. 22-36.

7. FERREIRA HA, et al. influência de agentes clareadores nas propriedades superficiais (rugosidade e microdureza) de uma cerâmica odontológica, cerâmica. 2016; P. 55-59.

8. GARCIA LFR, et al. Análise crítica do histórico e desenvolvimento das cerâmicas odontológicas, Rev Gaúcha Odontol. São Paulo. 2011; p. 67-73.

9. GRACIS S, et al. A New Classification System for All-Ceramic and Ceramic-like Restorative Materials, The Int J Prosthod. 2015; p.227-235.

10. HELVEY GA. Classifying Dental Ceramics: Numerous Materials and Formulations Available for Indirect Restorations, Compendium. 2014; p.38-43

11. CALAMIA JR, CALAMIA CS. Porcelain laminate veneers: reason for 25 years of success, Dent.Clín.N Am, 51, 2 2007; p. 399-417.

12. KRISHNA JV, et al. Evolution of metal-free ceramics, The Journal of Indian Prosthodontic Society, India, v.9, 2009; p. 70-75.

13. MAGNO P, BELSER U. Restaurações adesivas de porcelana na dentição anterior- Uma abordagem biomimética, $1^{a}$ ed. Quintessense Editora Ltda., São Paulo, 2012; p.406.

14. MATTEI FP, ALEXANDRE MC. Chain, Estado da arte das cerâmicas odontológicas. FULL Dentristry in Science, 2011; p. 84-91.

15. MCLAREN EA, FIGUEIRA J. Updating classifications of ceramic dental materials: a guide to material selection. Inside Dentistry. 2015; p. 48-72.

16. MCLAREN EA, FIGUEIRA J. Updating Classifications of Ceramic Dental Materials: A Guide to Material Selection, Compendium, 2015; p.400-406.

17. NAUTIYAL A, et al. Recent ceramic materials: an esthetic promise to patient, The Journal of Indian Prosthodontic Society.2015 p.26-30. 
18. SHRIVASTAVA S, et al. Ceramic cementation: a key to succesfull restoration, Annals and Essences of Dentistry. Department of Prosthodontics, Crown Bridge and Implantology. India, 2014; p. 35-43.

19. SILVA W, et al. Restabelecimento estético e funcional multidisciplinar, Full Dent. Sci. 2015; P. 210-219.

20. SKRIPNIK NN. Cerâmicas para facetas em dentes anteriores: uma revisão de literatura, TCC UFSC, 2016 ; P. 36p.

21. TRINDADE AC, et al. Quando dois se tornam um, PróteseNews, 3, v.4. 2016; p.394-399.

22. VARGAS MA. Cementing all-ceramic restorations, JADA, 2011; p. 205-245

23. VENTURINI AB, et al. Effect of Hydrofluoric Acid Concentration on Resin Adhesion to a Feldspathic Ceramic, J Adhes Dent. 2015; p. 313-320. 